\title{
Abduction in Observational and in Theoretical Sciences. Some Examples of IBE in Palaeontology and in Cosmology 1
}

\section{(La abducción en las ciencias observacionales y teóricas. Algunos ejemplos de IBE en paleontología y cosmología)}

\author{
Andrés Rivadulla RodRíGUEZ
}

Recibido: 25 de marzo de 2014

Aceptado: 23 de abril de 2015

\section{Resumen}

Contrariamente a la opinión de muchos filósofos de la ciencia de que ésta aplica el test deductivo de hipótesis, las ciencias observacionales de la Naturaleza, como la paleoantropología y las ciencias de la Tierra, emplean una metodología científica consistente en la propuesta de hipótesis que mejor se ajusten a los datos empíricos disponibles, e. d. que mejor expliquen los hechos. Las ciencias observacionales de la Naturaleza son predominantemente empíricas. Se basan en la observación y no hacen uso de tests popperianos deductivos de hipótesis. Las ciencias teóricas de la Naturaleza, como la física matemática, también aplican la inferencia a la mejor explicación para la introducción de conceptos e hipótesis relevantes. El objetivo de este artículo es mostrar que en la ciencia contemporánea el uso del razonamiento abductivo continúa siendo tan efectivo como siempre.

Palabras clave: abducción, ciencias observacionales, ciencias teóricas, paleoantropología, cosmología.

\footnotetext{
1 Complutense research group 930174 and research project FFI2014-52224-P on Natural Kinds financed by the Spanish Ministry of Economy and Competitiveness.
} 


\begin{abstract}
Contrary to the view maintained by many philosophers that science employs the deductive testing of hypotheses, observational natural sciences such as paleoanthropology and the earth sciences apply a scientific methodology consisting in the proposal of hypotheses which are best fitted to the available empirical data, i.e. which best explain the data. Observational natural sciences are predominantly empirical. They are grounded in observation, and they do not implement any Popperian deductive testing of hypotheses. Theoretical natural sciences such as mathematical physics also apply inference to the best explanation for the introduction of significant concepts and hypotheses. The aim of this paper is to demonstrate that in contemporary science, the use of abductive reasoning continues to be as effective as ever.
\end{abstract}

Keywords: abduction, observational sciences, theoretical sciences, paleoanthropology, cosmology

\title{
1. Introduction
}

Contrary to the view maintained by many philosophers that science makes use of the deductive testing of hypotheses, paleoanthropologists apply a scientific methodology consisting in the proposal of hypotheses which best fit to the available empirical data, i.e. which best explain the data. For this form of scientific inference, there exists a relatively old term: abduction.

Abduction was introduced by Charles S. Peirce, in his Collected Papers (CP, 5.171), as "the process of forming an explanatory hypothesis. It is the only logical operation which introduces any new idea". And in (CP, 5.145) he claims that "Abduction consists in studying facts and devising a theory to explain them." Norwood Russell Hanson (1958: 86) follows him in this. Since the 1980s, philosophers of science have been vindicating the role assumed by abductive reasoning in the methodology of science. In this way they are contributing to the rehabilitation of the context of discovery in the philosophy of science.

To illustrate this viewpoint, let me offer some quotations by notable contemporary paleoanthropologists. In a paper on the comparison of Neanderthal and modern human genomes, Svante Pääbo et al. (2006) claim that "Our knowledge of Neanderthals is based on a limited number of remains and artefacts from which we must make inferences about their biology, behavior, and relationship to ourselves." For his part Carlos Lorenzo (2005: 103) affirms that "Philogenetic trees are only evolutionary hypotheses built upon a continuously changing empirical basis. It is 
usual that these hypotheses are tested, and modified, if necessary, on the ground of new data." And in a similar way, Juan Luis Arsuaga and Ignacio Martínez (2006: 361) assert that "The main reason that contradictory hypotheses may be held on the origin of modern humans lies in nothing other than in the nature of the fossil register. Palaeontologists are seeking to reveal a process which took place over hundreds of thousands of years in three continents and which affected thousands of individuals. In order to undertake this work they have only a handful of fossils, usually very fragmentary, isolated and widely spread over time and space. There can be no doubt that the gaps in the register are considerably larger than the features we know about."

In order to do justice to this fact in the methodology of science, Rivadulla (2010) has introduced the distinction between observational and theoretical sciences in the realm of natural sciences. Observational natural sciences are predominantly empirical. They are grounded in observation, they apply abduction or inference to the best explanation (IBE) as a discovery practice, and they do not implement any Popperian deductive testing of hypotheses. For their part, theoretical natural sciences rely both on abduction and preduction ${ }^{2}$ for the introduction of novel hypotheses in the context of discovery, and they make use of the testing of theories in the context of justification. A typical example of theoretical natural science is mathematical physics. Paradigmatic examples of observational natural sciences are Paleoanthropology and the Earth sciences.

Paul Thagard (1978: 77) claims that "Inference to scientific hypothesis on the basis of what they explain was discussed by such nineteenth-century thinkers as William Whewell and C. S. Peirce, ... To put it briefly, inference to the best explanation consists in accepting a hypothesis on the grounds that it provides a better explanation of the evidence than is provided by alternative hypotheses."3 My aim in this paper is to show through some examples from contemporary science that nowadays the use of abductive reasoning is as effective as it was in the times of Peirce and Whewell.

\footnotetext{
2 Theoretical preduction, or simply preduction, is the name I give to the creative deductive practice in theoretical natural sciences like physics. I have defended the role of preductive reasoning in the context of scientific discovery in Rivadulla (2008, 2010 and 2015).

3 I identify Peircean abduction with inference to the best explanation as well. In this I follow Gilbert Harman (1965: 88-89) who introduced for the first time this equivalence, followed in his proposal by many contemporary methodologists of science. Even at the meta-methodological level, the defence of scientific realism grounds on an abductive argument: realism provides allegedly the best explanation for the empirical success of science.

From a logical point of view, Atocha Aliseda (2006 and 2014) and the Sevilla Group of Logic, Language and Information: Ángel Nepomuceno, Enrique Sarrión, Francisco J. Salguero, Ignacio Antón, among many others, have made relevant contributions to the understanding of abductive inference.
} 


\section{Human evolution. A surprising discovery at the Sima de los Huesos, Atapuerca, Spain}

On December 5, 2013, a team of researchers made up of the Spanish paleoanthropologists overseeing the excavations of the Atapuerca paleontological deposits, together with scientists from the Max Planck Institute for Evolutionary Anthropology, published in the journal Nature an article in which they presented an extraordinarily surprising discovery based on the mtDNA (mitochondrial DNA) analysis of Femur XIII, found in two excavations in 1994 and in 1999 at the Sima de los Huesos. The mitochondrial genomic sequence of the individuals inhabiting the Sima de los Huesos four hundred thousand years ago is closely related to the mtDNA of Homo denisovanus, a population of individuals from Southeast Siberia that lived in geologically relatively recent times. Indeed $H$. denisovanus lived at the same time that Neanderthals and Sapiens did. Arsuaga et al. (2013) present the discovery, the results of detailed mtDNA analysis, and the hypotheses that could best explain the discovery itself.

1.95 grams were extracted from Femur XIII. This provided the material basis for the mtDNA analysis. The reason why they decided to sequence the mtDNA is that the mtDNA was less deteriorated than the nuclear DNA. The results of the analysis were compared with the Denisovans' mtDNA which had been known since 2010. Mitochondrial DNA is only transmitted by women. Thus, if a woman does not bear a girl, the transmission of this DNA collapses for ever.

According to Arsuaga et al. (2013), "The fact that the Sima de los Huesos mtDNA shares a common ancestor with Denisovan rather than Neanderthal mtDNAs is unexpected 4 in light of the fact that the Sima de los Huesos fossils carry Neanderthal-derived features." The 400,000 year old Sima de los Huesos population, of which there is available a fossil register of 28 individuals, is philogenetically related with Denisovans, a population that lived in Southeast Siberia 40,000 years ago, and not to Neanderthals, as it was expected due to the fact that they share anatomic features in their dental, mandibular, midfacial, supraorbital and occipital morphology. As Ewen Callaway (2013) claims, experts are baffled. And as Arsuaga et al. (2013) affirm in the last paragraph of their article "the mtDNA sequence establishes an unexpected link between Denisovans and the western European Middle Pleistocene fossil record."

In order to explain this surprising fact, experts abductively infer four possible evolutionary scenarios, of which the first two can easily be excluded. In the first place, Arsuaga et al. (2013) guess that the Sima de los Huesos hominins might be closely related to the Denisovans's ancestors. Nonetheless this hypothesis seems

\footnotetext{
4 Unexpected or surprising facts trigger abductive inferences, i. e. inferences to the best explanation, as Charles Peirce and the subsequent philosophical literature on the methodology of abduction have been claiming.
} 
improbable "because the presence of Denisovans in Western Europe would indicate an extensive spatial overlap with Neanderthal ancestors, raising the question how the two groups could genetically diverge while overlapping in range. Furthermore... the Sima de los Huesos specimen is so old that it probably predates the population split between Denisovans and Neanderthals." The second hypothesis is that "the Sima de los Huesos hominins represent a group distinct from both Neanderthals and Denisovans that later perhaps contributed the mtDNA to Denisovans." However, the experts suspect that "this scenario would imply the independent emergence of several Neanderthal-like morphological features in a group unrelated to Neanderthals." The third possibility is that "the Sima de los Huesos hominins may be related to the population ancestral to both Neanderthals and Denisovans. Considering the age of the Sima de los Huesos remains and their incipient Neanderthal-like morphology, this scenario seems plausible to us, but it requires an explanation for the presence of two deeply divergent mtDNA lineages in the same archaic group, one that later recurred in Denisovans and one that became fixed in Neanderthals." Finally, the fourth possible scenario is that "gene flow from another hominin population brought the Denisova-like mtDNA into the Sima de los Huesos population or its ancestors. Such a hominin group might have also contributed mtDNA to the Denisovans in Asia." Following, "more than one evolutionary lineage may have existed in Europe during the Middle Pleistocene."

Arsuaga (2013) guesses that the most reliable hypothesis is that "at the time of the Sima de los Huesos, several individuals (the Femur XIII to begin with) and even some family groups did carry a non-Neanderthal archaic mitochondrial genome that disappeared, leaving behind only the Neanderthal genome. ... But it is also plausible that more than one human type lived and evolved in Europe and part of Asia: the ancestors of Neanderthals and 'the others', and that they cross-bred with each other. This is how an ancient mitochondrial genome would reach both the Sima population and the ancestors of the Denisovans."

Quite who the 'others' were, is the question Arsuaga leaves open in his commentary.

\section{Theoretical physics. Two Amazing discoveries in astrophysics and cosmology}

\subsection{Dark matter}

The Dutch astrophysicist Jan Hendrik Oort (1900-1992), who lends his name to the comet region around the solar system, calculated that our distance from the cen-

\footnotetext{
${ }^{5}$ Cf. Rivadulla (2003: 155 y ss.).
} 
tre of our galaxy amounts to 30,000 light years, and that our galaxy has approximately one hundred thousand million stars. Moreover, as in 1932 he studied the Doppler effect 5 in the spectra of some stars of the Galaxy, he found out that these stars did move more quickly than it was expected that they should. A year later, the Swiss astronomer Fritz Zwicky (1898-1974), measured the velocities in the galaxies of Coma Cumulus and observed the same phenomenon. The best explanation they came up with for these surprising facts was that in the galaxies there may be much more hidden than observable matter. Zwicky called it dark matter.

In order to tackle this problem from a theoretical standpoint, astrophysicists resort to Kepler's Third Law $P^{2} \propto D^{3}$. Since $D / P$ denotes for instance the orbital velocity of a star around its galaxy centre, it results that $v_{\text {orb }} \approx \sqrt{1 / D}$. Or, what amounts to nearly the same: $v_{\text {orb }} \propto D^{-1}$. According to this result, the orbital velocity of stars located far away from their galaxy centre is inversely related to their distance to the centre. Thus, it should decline with distance: the larger the distance, the smaller the star orbital velocity.

In order to find out the circular rotation velocity of any star around its galaxy centre, astronomers build so-called galactic rotation curves. During the 1970s, Vera Rubin and Ken Ford, among other astrophysicists, unexpectedly discovered that the rotation velocities of stars situated far away from their respective galactic centres remained nearly flat, i.e. parallel to the abscissas axis that represents the distances - measured in kilo-parsecs 6 - to the galactic centre. Thus these observations reveal that, at a great distance from the galactic centre, the orbital speed of stars is approximately constant, i.e. that it does not slow down by the amount anticipated by the application of Kepler's Third Law.

Instead of concluding that Kepler's Law is wrong, astrophysicists argue the following: "a flat rotation curve $V(R)=$ constant corresponds to a linear increase of mass with distance: $M(R) \propto R$. This means that the mass in the sphere interior of radius $R$ must continue to increase for large distances to the nucleus, but this mass cannot be observed. For this reason, the discovery that rotation curves are flat implies that dark matter must exist in the halos of spiral galaxies (...) i.e. non-luminous matter, which cannot be detected by means of telescopes. The gravitational action of this matter is responsible for the rotation curves becoming flat." (Cf. Vicent J. Martínez 2004: 225-227)

This is a splendid example of an Abductive Inference, an Inference to the Best Explanation. Moreover the dark matter hypothesis, which from a methodological point of view is an ad hoc hypothesis intended to save Newtonian mechanics from refutation, contributes to an increase in the falsifiability of this theory. Indeed, a hypothesized increase of mass - remember that it is not directly observed - impels the observed matter to move faster. And this obliges astrophysicists to search for it.

61 parsec (pc) is approximately 3,26 light years. 
Many physicists today are hunting for dark matter. According to Samuel Ting7, the main researcher of the AMS (Alpha Magnetic Spectrometer) project, this instrument, launched by the Endeavour on May 16, 2011, and coupled to the International Space Station, has detected a continuous flow of positrons possibly resulting from collisions between dark matter particles. Nonetheless scientists do not exclude that the positrons might proceed from pulsars as well. What will decide the issue is that the energy of positrons proceeding from collisions of dark matter particles has an upper limit. More data is still needed in order to reach any conclusion.

It is assumed that dark matter is largely non baryonic 8 , with the most likely candidates being Weakly Interacting Massive Particles (WIMP). The existence of dark matter has serious consequences for the fate of the Universe. Indeed if there were dark matter alone to be added to the ordinary baryonic matter, then the Universe would tend to collapse because of gravitational interaction. Again surprisingly, available observations support the hypothesis that the Universe is not only expanding, but that it is doing so in an accelerated way.

\subsection{Accelerated Expansion of the Universe and Dark Energy}

The expansion of the Universe is a theoretical possibility already envisaged by special theory of relativity. The first confirmations of this phenomenon were made by Vesto Melvin Slipher and Edwin Powell Hubble in the first quarter of the twentieth century. But in 1998, observations of supernovae of type A (Ia class), situated $4300 \mathrm{Mpc}$ (mega parsec) away from us, show that they are more distant than would be expected if the Universe were to contain only matter, since the gravitational attraction would slow down its expansion. These observations thus suggest that the Universe is not only expanding, but that it is also accelerating. In order to provide an explanation for this unexpected phenomenon, physicists proposed the hypothesis of the existence of some dark energy. For this discovery Saul Perlmutter, Brian Schmidt and Adam Riess were awarded the Nobel Physics Prize in 2011.

In declarations to the Spanish journal El País, published on 28th April 2013, Brian Schmidt declared that "The cosmological constant, that Einstein called his biggest error, could be my major discovery." Honouring Einstein, he claimed that "There are many alternative explanations, thousands of them, but Einstein's cosmological constant is the best and the most simple one." And in agreement with Popper, whom he does not mention, Schmidt affirms that "any scientist who is exploring at the frontiers of knowledge, entering the new, must dare to commit errors."

\footnotetext{
7 Information from spaceflight.com/news, April 5th 2013.

8 Baryonic matter is ordinary matter, i. e. matter made of quarks, for instance protons and neutrons.
} 
The postulation of the dark energy hypothesis, until now a completely unknown form of energy - hence the name dark energy - which is associated to Einstein's cosmological constant, is certainly an Inference to the Best Explanation, as it is the hypothetical proposal following an abductive inference.

Nonetheless the formalism of relativity theory again - general relativity in this case - admits of the possibility of the accelerating expansion of the Universe. Indeed, let us assume a galaxy that for convenience has mass unity, which is situated at the border of the Universe. Friedmann's equations of the general relativity theory (Cf. Rivadulla 2003: 237)

$$
\frac{\ddot{R}}{R}=-\frac{8 \pi G}{3} \rho+\frac{\Lambda}{3},
$$

allow us to deduce, substituting for $\rho$, the Universe's density, its value in $M=\frac{4}{3} \pi R^{3} \rho$, the mass of an spherical Universe, that

$$
\ddot{R}=-\frac{2 G M}{R^{2}}+\frac{\Lambda}{3} R,
$$

where $\ddot{R}$ is an acceleration, $R$ is the 'radius' of the Universe, $G$ is Newton's constant, and $\Lambda$ is Einstein's cosmological constant. Taking out numbers for simplicity, if we now take $m$ to denote the mass of the galaxy and multiply by it in both sides of the equation, we obtain the expression of the force acting upon the galaxy:

$$
m \frac{d^{2} R}{d t^{2}}=-G \frac{M m}{R^{2}}+m \Lambda(R) .
$$

The first summand at the right side of the equation is Newton's gravitation law, expressing the force acting upon the galaxy; it is negative, and this means that it is attractive. The second summand shows that the galaxy is also submitted to the action of a repulsive force - it is positive - associated to Einstein's cosmological constant. Since, as the sign of the left member of the equation shows, the resultant of the addition of both forces is positive, then the repulsive force is dominant and the galaxy of mass $m$, which was assumed to be located at the border of the Universe, is accelerating. Consequently the Universe itself is accelerating. As cosmologists have no idea about the nature of the repulsive force, associated to Einstein's cosmological constant, which is accelerating the Universe's expansion, they call it dark energy.

According to the Cosmological Standard Model, dark energy would contribute nearly $73 \%$ of the matter/energy in the Universe, dark matter $23 \%$, and only $4 \%$ ordinary, basically baryonic, matter. 


\section{Conclusion}

Most twentieth century thinkers have neglected abduction as a result of their rejection of the relevance of the context of discovery for the philosophy of science. The development of Western science shows that this was a major error. Moreover, as this paper argues, natural sciences such as palaeoanthropology and theoretical physics nowadays continue to employ abductive reasoning, inference to the best explanation, in order to introduce new ideas into science.

\section{References}

AlisedA, A. (2006): Abductive Reasoning. Logical Investigations into Discovery and Explanation. Dordrecht: Springer

AlisedA, A. (2014): La lógica como herramienta de la razón. Razonamiento ampliativo en la creatividad, la cognición y la inferencia. Cuadernos de Lógica, Epistemología y Leguaje, Vol. 6. College Publications. Milton Keynes, Reino Unido.

Arsuaga, J. L. y Martínez, I. (2006): La especie elegida. La larga marcha de la evolución humana. Madrid: Temas de Hoy

Arsuaga, J. L. (2013): "Los otros". El País. Sociedad, December 4th.

Arsuaga, J. L.; Martínez, I.; Bermúdez de Castro, J. M; Carbonell, E.; Gracia, A.; Meyer, M.; PÄÄBo, S. (et al.) (2013): "A mitochondrial genome sequence of a hominin from Sima de los Huesos", Nature, vol. 504, Letter.

Callaway, E. (2013): "Hominin DNA baffles experts. Analysis of oldest sequence from a human ancestor suggests link to mystery population". Nature, vol. 504

Lorenzo, C. (2005): "Primeros homínidos. Géneros y especies". In Eudald Carbonell (ed.), Homínidos: Las primeras ocupaciones de los continentes. Barcelona: Ariel

Hanson, N. R. (1958): Patterns of Discovery. An inquiry into the conceptual foundations of science. Cambridge: University Press

Harman, G. (1965): "The Inference to the Best Explanation". The Philosophical Review, vol. 74, No. 1: 88-95

Hernández Antón, I.; Nepomuceno, A. y SARrión, E. (2012): "La inferencia relativa al contexto" En L. Fernández, F. J. Salguero y C. Barés (eds.), Ensayos sobre Lógica, Lenguaje, Mente y Ciencia. Sevilla: Ediciones Alfar

PÄÄвO, S. et al. (2006): "Sequencing and Analysis of Neanderthal Genomic DNA", Science, Vol. 314

Peirce, C. S. (1965): Collected Papers. Cambridge, MA: Harvard University Press Rivadulla, A. (2003): Revoluciones en Física. Madrid: Trotta 
Rivadulla, A. (2008): "Discovery Practices in Natural Sciences: From Analogy to Preduction". Revista de Filosofía, Vol. 33: 117-137

Rivadulla, A. (2010): "Complementary Strategies in Scientific Discovery: Abduction and Preduction". In Bergman, M.; Paavola, S.; Pietarinen, A.-V. \& Rydenfelt, H. (Eds.), Ideas in Action. Proceedings of the Applying Peirce Conference. Nordic Studies in Pragmatism 1. Helsinki: Nordic Pragmatism Network, 241-253.

Rivadulla, A. (2015): Meta, Método y Mito en Ciencia. Madrid: Trotta.

Smolin, L. (2007), The Trouble with Physics. The Rise of String Theory, the Fall of a Science, and What Comes Next. Boston-New York: Houghton Mifflin Co.

Thagard, P. R. (1978): "The Best Explanation: Criteria for Theory Choice". The Journal of Philosophy, vol 75, № 2: 76-92.

Andrés Rivadulla Rodríguez

Departamento de Lógica y Filosofía de la Ciencia

Universidad Complutense de Madrid arivadulla@filos.ucm.es 